\title{
SPRAY DRYING DE DISPERSÕES LIPÍDICAS CONTENDO ANTIOXIDANTES NATURAIS
}

\author{
I. BALDIM, W.P. OLIVEIRA, C.R.F. SOUZA* \\ Universidade de São Paulo, Faculdade de Ciências Farmacêuticas de Ribeirão Preto, Laboratório de \\ P\&D em Processos Farmacêuticos - LAPROFAR. \\ *e-mail: souzacrf@gmail.com
}

\begin{abstract}
RESUMO
O alecrim (Rosmarinus officinalis) é uma fonte de compostos antioxidantes que podem contribuir na prevenção de doenças degenerativas (câncer e diabetes). No entanto, instabilidades como a auto-oxidação e degradação desses compostos reduzem sua eficácia. Portanto, existe a necessidade de desenvolver sistemas que melhorem a estabilidade de seus compostos bioativos. Os carreadores lipídicos são efetivos sistemas de encapsulação, apresentando facilidade de preparo, escalonamento e baixa toxicidade. O objetivo deste trabalho foi produzir sistemas micro e nanoestruturados através do emprego de carreadores lipídicos contendo extrato liofilizado de alecrim e avaliar a influência dos tipos e proporções de adjuvantes na secagem por spray dryer. Os parâmetros de secagem foram temperatura do ar de entrada: $100^{\circ} \mathrm{C}$, vazão do ar de secagem: $60 \mathrm{~m}^{3} / \mathrm{h}$, vazão de alimentação: $4 \mathrm{~g} / \mathrm{min}$ e pressão do ar de atomização de 3 $\mathrm{kgf} / \mathrm{cm}^{2}$. O rendimento do processo manteve-se entre 28 e $67 \%$, com teor de umidade abaixo de $6 \%$ e atividade de água de 0,3 . A análise de tamanho de partícula forneceu valores em torno de $900 \mathrm{~nm}$, sendo os pós obtidos classificados como de baixo fluxo.
\end{abstract}

\section{INTRODUÇÃO}

Plantas medicinais e aromáticas são importantes fontes de compostos bioativos de elevado valor terapêutico e nutricional. $\mathrm{O}$ alecrim (Rosmarinus officinalis Linné) é uma planta muito utilizada como flavorizante de alimentos devido às suas propriedades aromáticas. Também é utilizada como planta medicinal, apresentando comprovadas propriedades antioxidante, hepatoprotetora, antidiabética, antimicrobiana, antiproliferativa, dentre outras (ALMELA et al., 2006; SOUZA et al., 2008).

Devido à elevada atividade antioxidante do alecrim, suas preparações são utilizadas em várias aplicações como: preservante de alimentos, cosméticos, nutracêuticos e como fitoterápico (COUTO et al., 2012). Sua atividade antioxidante está associada à presença de compostos fenólicos, como ácido carnósico e carnosol e a flavonoides (ALMELA et al., 2006; GENENA, 2005). Porém, esses compostos, principalmente o ácido carnósico e o carnosol, são susceptíveis à degradação (ZHANG et al., 2012). O ácido carnósico é o diterpeno fenólico encontrado em maior quantidade em folhas frescas de alecrim, porém durante $\mathrm{o}$ processo de extração este é convertido em carnosol ou outros diterpenos (GENENA, 2005). Assim, a manutenção destes compostos nos extratos vegetais e produtos tecnológicos sofre influência das condições de preparação, incluindo condições de extração e estocagem e processos de produção de sistemas micro e nanoestruturados. 
Dentre as estratégias que podem ser empregadas na obtenção de sistemas particulados contento produtos naturais incluem-se a micro/nanoencapsulação. Micro e nanoencapsulação são definidos como os processos em que pequenas partículas ou gotículas são revestidas por um material de parede ou embebidas em uma matriz homogênea ou heterogênea para a obtenção de pequenas cápsulas, o que cria uma barreira entre os compostos de dentro da cápsula e o ambiente (CALVO et al., 2012).

A escolha desse material vai depender de sua capacidade em conferir proteção e o aumento da estabilidade ao princípio ativo. Os carreadores lipídicos são citados nas mais diversas aplicações devido a suas propriedades únicas, como a capacidade de aumentar a solubilidade, facilidade em incorporar compostos tanto lipossolúveis como hidrossolúveis, melhoria da biodisponibilidade in vivo e a estabilidade do ativo (GORTZI et al., 2008).

Os produtos em estado líquido são mais instáveis que as respectivas formas sólidas. Assim, a secagem do sistema permite aumentar sua estabilidade e o tempo de armazenamento do produto. Operações como liofilização, secagem por spray drying ou spray freeze drying, entre outros, produzem sistemas na forma de pós, capazes de aumentar a estabilidade e superar os problemas de degradação pela correta escolha das variáveis de processo e composição da formulação de encapsulamento (COURRIER, BUTZ, ADAMME, 2002).

O objetivo deste trabalho foi a produção de sistemas micro e nanoestruturados, contendo extrato liofilizado de alecrim, e avaliação da influência dos tipos e proporções de adjuvantes na secagem por spray dryer e a caracterização físico-química do produto.

\section{MATERIAIS E MÉTODOS}

\subsection{Matéria-prima vegetal}

A matéria-prima vegetal, constituída por folhas secas e moídas de Rosmarinus officinalis, foi adquirida do grupo Santos Flora (São Paulo - SP).

\subsection{Lipídeos e carreadores de estabilização e secagem}

Os lipídeos selecionados para o desenvolvimento deste trabalho foram a fosfatidilcolina de soja hidrogenada $90 \%$ (Phospholipon ${ }^{\circledR} 90 \mathrm{H}, \quad$ Lipoid $\mathrm{GMBH}$, Ludwigshafen, Alemanha) e o colesterol 94\% (Sigma-Aldrich, St. Louis, MO, USA).

A adição de carreadores de secagem visa melhorar o desempenho do processo, auxiliar na proteção dos compostos bioativos e melhorar a qualidade físico-química do produto seco. Assim, neste estudo foram avaliados como carreadores de secagem a lactose M-200 (Natural Pharma Produtos Farmacêuticos Ltda, São Paulo, SP, Brasil) e o Lacprodan - concentrado proteico de soro de leite (Arla Foods Ingredients S.A., Porteña, Argentina).

\subsection{Preparo do extrato concentrado e liofilizado}

A solução extrativa foi preparada por maceração dinâmica, tendo como solvente extrator uma solução de etanol $70 \%$ (v/v), proporção de $1: 10(\mathrm{p} / \mathrm{v})$ planta/solvente e temperatura de extração de $70{ }^{\circ} \mathrm{C}$, por 60 minutos. Após a obtenção da solução extrativa esta foi concentrada em evaporador rotativo (Fisatom modelo 802), a temperatura de $50{ }^{\circ} \mathrm{C}$ e vácuo de $600 \mathrm{mmHg}$.

$\mathrm{O}$ extrato concentrado foi submetido ao processo de liofilização (extrato liofilizado), em liofilizador VLP 195 FD-115, Thermo Fischer Scientific, temperatura de condensação de $-40{ }^{\circ} \mathrm{C}$ por 48 h. O extrato obtido foi acondicionado em recipiente hermético à temperatura de $-20{ }^{\circ} \mathrm{C}$. 


\subsection{Preparo das dispersões lipídicas}

As dispersões lipídicas contendo $R$. officinalis foram preparadas pelo método adaptado de Lo, Tsai e Kuo (2004), e a formulação teve suas proporções fixadas (Tabela 1) de acordo com dados da literatura (SECOLIN; OLIVEIRA; SOUZA, 2014). O preparo das formulações é realizado em 2 fases distintas. A Fase 1 é composta pela água, o extrato liofilizado de $R$. officinalis, o carreador (lactose ou proteína) e a goma xantana. A Fase 2 é composta por água, álcool n-butílico, lecitina e colesterol. Ambas as fases são mantidas em placa de aquecimento sob agitação até atingirem $70^{\circ} \mathrm{C}$. Quando atingida a temperatura, verte-se a fase 1 lentamente sobre a fase 2 e homogeneíza-se a composição obtida em ultraturrax a 18000 rpm por 3 minutos. Para que as vesículas se estabilizem, mantém-se a formulação em agitação lenta por 2 horas a temperatura ambiente. A Figura 1 apresenta o aspecto visual das formulações obtidas.

Tabela 1 - Esquema de constituintes e proporção de adjuvantes das dispersões lipídicas contendo extrato liofilizado de $R$. officinalis.

\begin{tabular}{ccccccc}
\hline Componentes & F1L3 & F1P3 & F1LP3 & F1L6 & F1P6 & F1LP6 \\
\hline Lecitina $(\mathbf{g})$ & 4,0 & 4,0 & 4,0 & 4,0 & 4,0 & 4,0 \\
Colesterol $(\mathbf{g})$ & 0,5 & 0,5 & 0,5 & 0,5 & 0,5 & 0,5 \\
Extrato Liofilizado (g) & 2,0 & 2,0 & 2,0 & 2,0 & 2,0 & 2,0 \\
Lactose (g) & 3,0 & - & - & 6 & - & - \\
Proteína (g) & - & 3,0 & - & - & 6 & - \\
Lactose + Proteína (g) & - & - & $1,5: 1,5$ & - & - & $3,0: 3,0$ \\
Álcool n-butílico (ml) & 24,0 & 24,0 & 24,0 & 24,0 & 24,0 & 24,0 \\
Água (g) & 90,3 & 90,3 & 90,3 & 87,3 & 87,3 & 87,3 \\
Goma Xantana (g) & 0,2 & 0,2 & 0,2 & 0,2 & 0,2 & 0,2 \\
\hline
\end{tabular}

Figura 1 - Dispersões lipídicas contendo extrato liofilizado de $R$. officinalis.

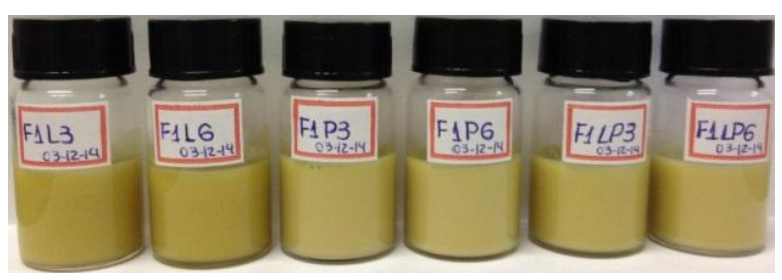

\subsection{Spray drying}

Utilizou-se um spray dryer escala laboratorial, modelo SD-05 fabricado pela Lab-Plant, Reino Unido, que possui uma câmara de secagem de $215 \mathrm{~mm}$ de diâmetro e $500 \mathrm{~mm}$ de altura. As dispersões lipídicas foram alimentadas a uma vazão $\left(\mathrm{W}_{\text {susp }}\right)$ de 4 $\mathrm{g} / \mathrm{min} \mathrm{e}$ as variáveis operacionais foram fixadas em: temperatura de ar de entrada $\left(\mathrm{T}_{\mathrm{ge}}\right)$ : $100^{\circ} \mathrm{C}$, vazão do ar de secagem $\left(\mathrm{W}_{\mathrm{g}}\right)$ : $60 \mathrm{~m}^{3} / \mathrm{h}$, pressão do ar de atomização $\left(\mathrm{P}_{\mathrm{atm}}\right): 3$ $\mathrm{kgf} / \mathrm{cm}^{2}$ e vazão do ar de atomização $\left(\mathrm{W}_{\mathrm{atm}}\right)$ : $17 \mathrm{lpm}$. A temperatura do gás de saída foi monitorada em intervalos regulares, de forma a se obter o instante em que o processo entra em regime permanente, iniciando-se a alimentação da formulação de secagem e a coleta do produto seco. Monitoraram-se a temperatura e umidade do ar ambiente e temperatura do ar de saída de forma a verificar o desempenho do secador. 


\subsubsection{Recuperação do produto}

A recuperação do produto $(\mathrm{R})$ foi calculada por balanço de massas através da relação percentual entre a massa de pó total coletada e a massa de sólidos alimentada no spray dryer (CORTES-ROJAS; OLIVEIRA, 2012), de acordo com Equação 1.

$\mathrm{R}=\frac{\mathrm{M}_{\mathrm{c}} \times\left(1-\mathrm{X}_{\mathrm{p}}\right)}{\mathrm{W}_{\mathrm{s}} \times \mathrm{C}_{\mathrm{s}} \times \theta} \times 100$

Onde: $\mathrm{M}_{\mathrm{c}}$ : massa coletada $(\mathrm{g}) ; \mathrm{X}_{\mathrm{p}}$ : umidade $(\mathrm{g})$; $\mathrm{W}_{\mathrm{s}}$ : vazão de alimentação $(\mathrm{g} / \mathrm{min}) ; \mathrm{C}_{\mathrm{s}}$ : teor de sólidos da composição de secagem (g/g); $\theta$ : tempo de processo (min).

\subsection{Caracterização das dispersões lipídicas secas}

Os produtos secos foram caracterizados quanto ao teor de umidade (Karl Fischer), atividade de água, densidade aparente e de compactação e índices de fluidez (Índice de Carr e Fator de Hausner), distribuição granulométrica, análise do tamanho de partícula, determinação da solubilidade das partículas em água e teor dos principais compostos ativos por HPLC.

\subsubsection{Determinação do teor de umidade}

O teor de umidade do produto foi analisado por titulação pelo método de Karl Fischer. Utilizou-se o equipamento Methrom $870 \mathrm{KF}$ Titrino Plus procedendo-se com a titulação das amostras. Foram utilizadas amostras de aproximadamente $100 \mathrm{mg}$ para cada determinação. As medidas foram realizadas em triplicata e os resultados expressos em média (desvio padrão) (FERNANDES, 2013).

\subsubsection{Determinação da atividade de água}

$\mathrm{A}$ atividade de água ( $\mathrm{Aa}$ ) das amostras foi determinada em triplicata no equipamento Aqua Lab 4 TEV (Decagon, Devices) à temperatura de $25^{\circ} \mathrm{C}$, utilizando o sensor de ponto de orvalho.
2.6.3 Densidade aparente e de compactação dos pós e índices de compactação

Para essa determinação, um grama das dispersões lipídicas secas foi pesado e transferido para proveta de $10 \mathrm{~mL}$. A densidade aparente foi determinada pela razão entre o volume $(\mathrm{mL})$ ocupado pelo material particulado e a massa total pesada (g). Para a determinação da densidade de compactação, a amostra contida na proveta foi submetida a 1250 quedas em superfície lisa e altura de 3,0 $\pm 0,2 \mathrm{~mm}$ com o auxílio do equipamento Caleva ${ }^{\circledR}$ TDT II (Frankfurt, Germany). A densidade de compactação foi calculada pela razão entre a massa em gramas e o volume em mililitros ocupado pelo material particulado após a compactação (FERNANDES, 2013).

Os Índices de compactação dos pós foram determinados através do fator de Hausner (FH) e índice de Carr (IC). O valor de $\mathrm{FH}$ foi determinado pelo quociente entre a densidade de compactação e a densidade aparente da formulação seca (Equação 2), e o IC foi expresso pelo quociente da diferença das densidades aparente e de compactação pela densidade aparente. O IC foi expresso em porcentagem, multiplicando-se o valor obtido por 100, conforme apresentado na Equação 3 (CORTES-ROJAS; SOUZA; OLIVEIRA, 2014).

$$
\begin{aligned}
& \mathrm{RH}=\frac{\rho \mathrm{c}}{\rho \mathrm{a}} \\
& \mathrm{IC}=\frac{(\rho \mathrm{c}-\rho \mathrm{a})}{\rho \mathrm{c}} \times 100
\end{aligned}
$$

Onde: $\rho_{\mathrm{c}}$ é a densidade de compactação $\left(\mathrm{g} / \mathrm{cm}^{3}\right)$ e $\rho_{\mathrm{a}}$ é a densidade aparente $\left(\mathrm{g} / \mathrm{cm}^{3}\right)$.

\subsubsection{Distribuição granulométrica}

A distribuição granulométrica foi determinada dispersando-se uma amostra do pó em uma lâmina de vidro com a ajuda de um dispersor de pós. As amostras foram analisadas em microscópio óptico (Olympus ${ }^{\circledR}$ modelo BX60MIV) conectado a uma câmera 
digital (modelo 3.2.0, SPOT Insight). As imagens obtidas com aumento de 50 vezes foram analisadas pelo sistema de análise de imagens (Image Pro-Plus ${ }^{\circledR}$ ). Para a contagem das partículas selecionou-se o diâmetro médio de partícula de 0 a $200 \mu \mathrm{m}$. Para garantir a confiança e a repetibilidade do método foi avaliado um mínimo 1.000 partículas (CORTES-ROJAS; OLIVEIRA, 2012).

\subsubsection{Análise do tamanho de partícula}

As dispersões lipídicas secas foram ressuspensas em água na proporção de 1:25. Após as diluições, as amostras foram submetidas à agitação mecânica por 20 minutos e submetidas a processador ultrasonico Vibracell VC750 (SONICS, Newtown, USA) com a sonda Microtip 1/8" 630-0422 na amplitude de 30\% durante 30 segundos. As análises foram realizadas no equipamento light scattering (Beckman Coulter LS 13320).

2.6.6 Determinação da solubilidade das partículas em água

Utilizou-se o método de Eastman e Moore (1984), com modificações. Amostras de $0,5 \mathrm{~g}$ foram dispersas em $50 \mathrm{~mL}$ de água em agitação magnética por $10 \mathrm{~min} e$ centrifugada a $2330 \mathrm{rcf}$ por $5 \mathrm{~min}$. Alíquotas de $15 \mathrm{~mL}$ do sobrenadante foram secas em estufa a $105{ }^{\circ} \mathrm{C}$ até peso constante. A solubilidade das dispersões lipídicas em água foi calculada com base na massa inicial da amostra que foi solubilizada na alíquota de 15 $\mathrm{mL}$ do sobrenadante. O resultado foi expresso em porcentagem e calculado pela Equação 4.

$\%$ Solubilida de $=\frac{\mathrm{m}_{\text {residual }}}{\mathrm{V}_{\mathrm{al}}} \times \mathrm{V}_{0} \times \frac{1}{\mathrm{~m}_{\text {inicial }}} \times 100$

Onde: $\mathrm{m}_{\text {residual: }}$ massa do resíduo seco, $\mathrm{V}_{\mathrm{al}}$ : volume da alíquota, $\mathrm{V}_{\mathrm{o}}$ : volume inicial, $\mathrm{m}_{\text {inicial }}$ : massa inicial.

\subsection{Quantificação dos ativos das formulações por cromatografia (HPLC)}

Tanto as dispersões lipídicas líquidas quanto as secas foram analisadas por cromatografia líquida de alta eficiência (HPLC) para a quantificação dos principais marcadores bioativos presentes no alecrim. $\mathrm{O}$ extrato liofilizado também foi analisado, em dois períodos diferentes: após o preparo e após 3 meses armazenado a $-20^{\circ} \mathrm{C}$.

Os ativos quantificados através do método HPLC foram: ácido cafeico (ACF), ácido rosmarínico (AR), carnosol (C) e ácido carnósico (ACN). A metodologia analítica usada foi adaptada do método descrito por Wellwood e Cole (2004). Utilizou-se um cromatógrafo líquido de alta eficiência Shimadzu Prominence, equipado com bombas de alta pressão mod. LC-10 AD, amostrador automático mod. SIL-10ADVP, detector espectrofotométrico Photodiodo Array mod. SPD-M10A, controlado pelo software LC Solution. O equipamento foi equipado com coluna de fase reversa C-18 Shimadzu (250 x $4,6 \mathrm{~mm}$ d.i., $5 \mu \mathrm{m}$ ) termostatizada a temperatura de $30{ }^{\circ} \mathrm{C}$, usando pré-coluna C18. As fases móveis utilizadas foram $0,1 \%$ ácido fórmico em água (A) e acetonitrila (B). Os gradientes variam de $15-35 \%$ de $B$ durante 0-20 minutos e de 35-100\% de B durante 20-30 minutos, permanecendo por 5 minutos em $100 \%$ de B. O gradiente é reduzido de $100-15 \%$ de $\mathrm{B}$ de 35 a 37 minutos de análise, permanecendo por mais 5 minutos em $15 \%$ de $\mathrm{B}$, finalizando a análise em 42 minutos. As fases móveis foram filtradas $(0,45 \mu \mathrm{m}$, Millipore $)$ e degaseificadas em ultrassom durante 3 minutos antes do uso, sendo empregada vazão total de $1,2 \mathrm{ml} \cdot \mathrm{min}^{-1}$. $\mathrm{O}$ volume de injeção da amostra foi de $10 \mu \mathrm{L}$ e a detecção ocorre em 284 e $330 \mathrm{~nm}$. Os picos cromatográficos são identificados comparando seus tempos de retenção com os de padrões e analisando-se o perfil do espectro UV referente ao pico. A 
quantificação é feita por integração da área do pico em comparação com padrão externo.

As curvas analíticas foram confeccionadas utilizando soluções de padrões de ácido cafeico (ACF), ácido rosmarínico (AR), carnosol (C) e ácido carnósico (ACN) em diversas concentrações, seguindo-se a metodologia descrita anteriormente e validada segundo o "Guia para validação de métodos analíticos e bioanalíticos” (BRASIL, RE 899/2003).

\section{RESULTADOS E DISCUSSÃO}

\subsection{Avaliação do desempenho do processo de secagem}

O parâmetro utilizado para avaliar o desempenho do secador foi a recuperação do produto. Como apresentado na Figura 2, a média de recuperação manteve-se em $50 \pm$ $16 \%$, sendo os maiores resultado obtidos para as formulações F1P6 (67,3\%), F1LP6 $(66,1 \%)$ e F1L6 $(58,7 \%)$, e menor para F1P3 $(28,6 \%)$. A tendência de recuperação foi maior para as formulações contendo combinação de lactose e proteína como carreador de secagem. $O$ aumento na concentração de carreador na composição de secagem tende a causar um efeito positivo na recuperação de produto seco.

Figura 2 - Resultados de recuperação do produto obtido por spray dryer.

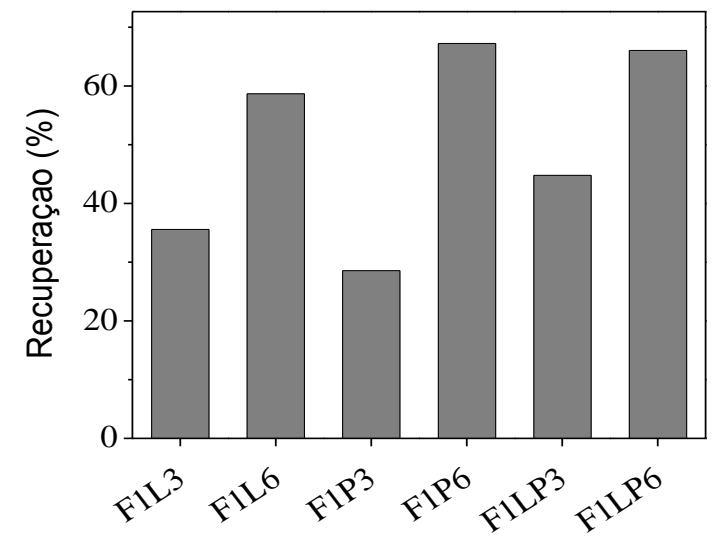

Tanto a lactose quanto o concentrado proteico de soro de leite são adjuvantes bastante utilizados industrialmente, devido à facilidade de obtenção e qualidade dos produtos gerados. Os valores de recuperação do produto obtidos neste trabalho (média $50 \pm$ $16 \%$ ) estão de acordo com os resultados encontrados em literatura para spray dryers de bancada, onde se verificam valores de recuperação na faixa de 30 a $80 \%$, dependente do tipo de material e configuração do secador (FERNANDES, 2013).

A Figura 3 apresenta o aspecto visual dos pós obtidos pelas secagens em spray dryer.

Figura 3 - Dispersões lipídicas contendo extrato liofilizado de $R$. officinalis secas por spray dryer.

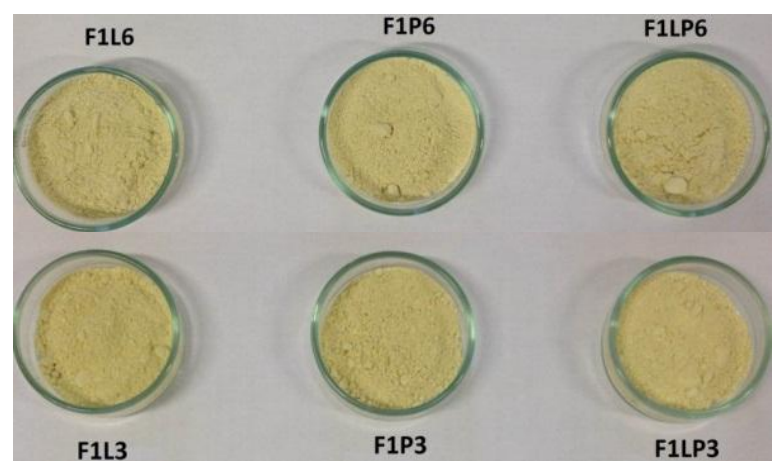

\subsection{Caracterização das micropartículas}

3.2.1 Teor de umidade e atividade de água

O teor de umidade é um importante indicador de segurança, estabilidade e qualidade de produtos secos. Os níveis de umidade podem influenciar em propriedades farmacotécnicas do produto como no grau de compressibilidade, a tendência de aglomeração das partículas, além de sua estabilidade (SOUZA; OLIVEIRA, 2012). Contudo, nem toda água presente no material está disponível para crescimento microbiano ou para a participação de reações químicas como a hidrólise. Em produtos secos níveis acima de 0,5 não são desejados por permitirem o crescimento microbiano e a ocorrência de reações de degradação. 
A caracterização do produto obtido pelo processo de secagem por spray drying mostrou um baixo teor de umidade $(<6 \%)$ e atividade da água inferior a 0,34 (Tabela 2).

Tabela 2 - Teor de umidade (Xp) e atividade de água (Aa) dos pós obtidos por spray dryer.

\begin{tabular}{ccc}
\hline Amostra & Xp ( \% ) & Aa $(-)$ \\
\hline F1L3 & $5,79(0,04)$ & $0,32(0,02)$ \\
F1L6 & $4,19(0,11)$ & $0,33(0,00)$ \\
F1LP3 & $2,72(0,04)$ & $0,24(0,04)$ \\
F1LP6 & $3,50(0,07)$ & $0,27(0,00)$ \\
F1P3 & $3,79(0,04)$ & $0,28(0,00)$ \\
F1P6 & $3,81(0,04)$ & $0,26(0,02)$ \\
\hline
\end{tabular}

As amostras contendo proteína em sua composição apresentaram valores de umidade e atividade de água inferiores àquelas contendo apenas lactose, o que pode estar relacionado à maior higroscopicidade da lactose quando comparada à proteína (Lacprodan ${ }^{\circledR}$ ). Todos os produtos apresentaram valores de atividade de água abaixo de 0,34 , sendo um fator favorável à manutenção da estabilidade dos produtos.

3.2.2 Densidade aparente e de compactação dos pós e índices de compactação

As dispersões lipídicas secas obtidas foram avaliadas quanto às suas propriedades farmacotécnicas através da determinação das densidades brutas e de compactação dos pós (Tabela 3).

A maior densidade de compactação obtida foi para o produto F1P3 e o maior índice de Carr para F1LP6. Produtos com valores de RH menor que 1,18 são classificados como de boa compressão. Em relação ao índice de Carr, materiais com índice entre 5 e $15 \%$ são considerados de fluidez excelente, de 12 a $16 \%$ fluxo bom, de 18 a $21 \%$ escassa, de 22 a $35 \%$ fluxo fraco, de 35 a $38 \%$ muito fraco e maior que $40 \%$ extremamente fraco (AULTON, 2005). Os resultados de RH e IC apresentados na Tabela 3 indicam que os produtos formados não apresentam boas propriedades de compressão, sendo classificados como de fluxo fraco e muito fraco.

Tabela 3 - Resultado de densidade aparente (Da), densidade de compactação (Dc), razão de Hausner (RH) e índice de Carr (IC) dos pós obtidos por spray dryer.

\begin{tabular}{ccccc}
\hline Formulação & $\begin{array}{c}\text { Da } \\
\left(\mathbf{g} / \mathbf{c m}^{\mathbf{3}}\right)\end{array}$ & $\begin{array}{c}\mathbf{D c} \\
\left(\mathbf{g} / \mathbf{c m}^{\mathbf{3}}\right)\end{array}$ & $\begin{array}{c}\text { RH } \\
(-)\end{array}$ & $\begin{array}{c}\text { IC } \\
(\mathbf{\%})\end{array}$ \\
\hline F1L3 & 0,300 & 0,375 & 1,3 & 25,0 \\
F1L6 & 0,314 & 0,406 & 1,3 & 29,6 \\
F1LP3 & 0,265 & 0,371 & 1,4 & 40,0 \\
F1LP6 & 0,261 & 0,375 & 1,4 & 44,0 \\
F1P3 & 0,365 & 0,510 & 1,4 & 40,0 \\
F1P6 & 0,210 & 0,292 & 1,4 & 39,3 \\
\hline
\end{tabular}

3.2.3 Distribuição granulométrica e tamanho de partícula

Os pós obtidos nas secagens por spray drying foram caracterizados quanto a distribuição granulométrica por microscópio óptico, pelo qual foram obtidas as frações acumuladas (Figura 4) e análise de imagem. e por espalhamento de luz pelo equipamento light scattering (Figura 5).

Figura 4 - Distribuição granulométrica acumulada das micropartículas obtidas.

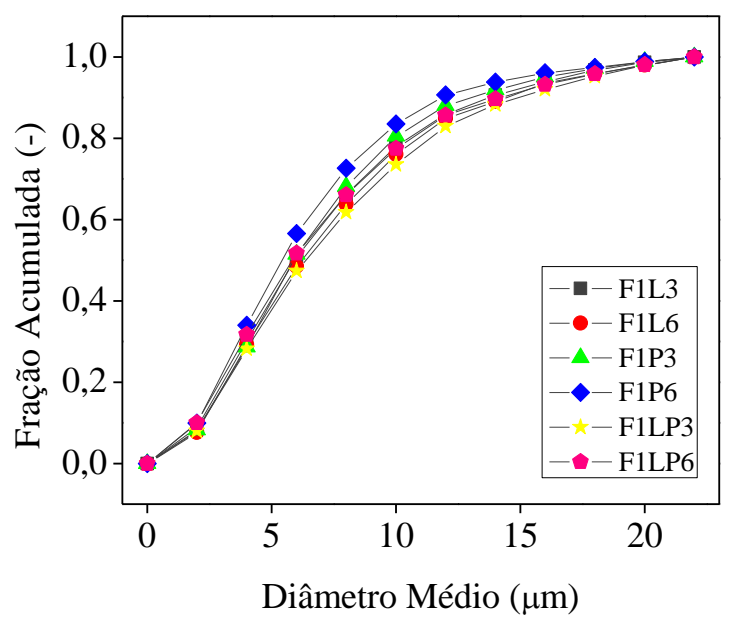


A Figura 5 apresenta os resultados da análise do tamanho de partícula das dispersões lipídicas originais e do produto seco redisperso em água, através da técnica de espalhamento de luz (light scattering). Houve pequena variação de tamanho das partículas para as dispersões lipídicas líquidas (antes do processo de secagem) e das formulações secas por spray drying redispersas em água, sendo os valores em torno de 850 a $900 \mathrm{~nm}$ para as dispersões líquidas e 810 a $920 \mathrm{~nm}$ para as amostras secas redispersas.

Figura 5 - Tamanho de partícula das dispersões lipídicas originais e do produto seco redisperso.

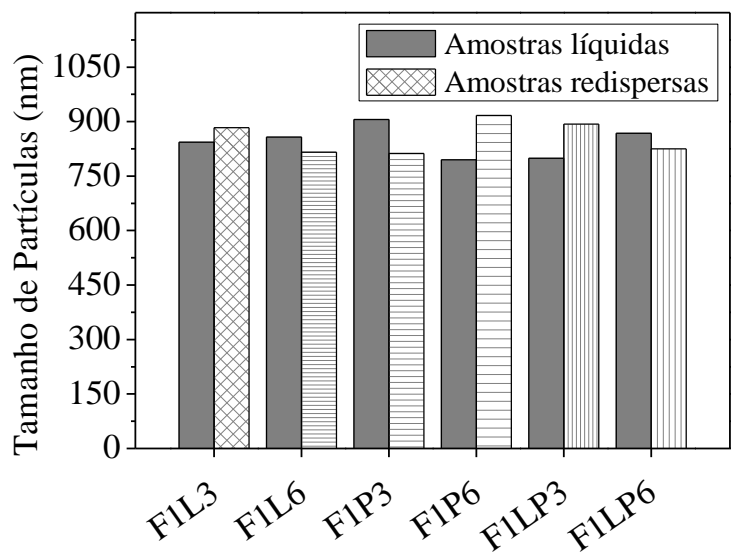

\subsubsection{Solubilidade das partículas em água}

Os resultados obtidos (Figura 6) indicam, em média, que todas as formulações lipídicas secas apresentaram uma baixa solubilidade (redispersão) em água, o que pode ser justificado parcialmente pelos constituintes lipídicos.

As amostras contendo lactose como carreador de secagem se mostraram mais solúveis em água (de acordo com a metodologia empregada), com valores de solubilidade de 45 e $60 \%$ (proporção de carreador lipídio 1:1 e 1:2, respectivamente). As formulações contendo proteína como carreador apresentaram valores médios de solubilidade de $30 \%$. Para as dispersões obtidas com misturas de lactose/proteína, os valores obtidos são de 35 e $45 \%$ (proporção de carreador lipídio 1:1 e 1:2, respectivamente), aumentando a solubilidade pelo aumento da proporção de carreador.

Figura 6 - Solubilidade das partículas em água.

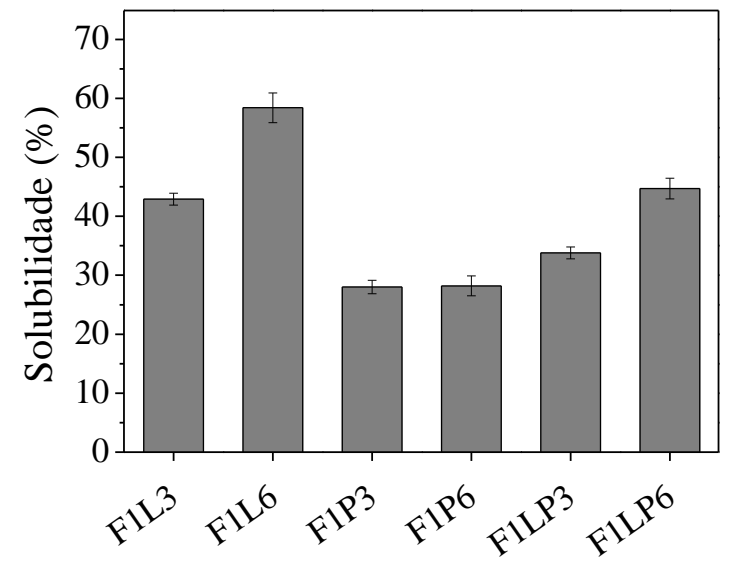

\subsection{Dosagem de ativos das formulações por cromatografia (HPLC)}

Com relação à dosagem de ativos (Figura 4) do extrato liofilizado, pode-se ressaltar que após 3 meses de armazenamento em freezer $\left(-20{ }^{\circ} \mathrm{C}\right) \quad \mathrm{o}$ extrato sofreu degradações significativas tanto de ácido cafeico, carnosol quanto de ácido carnósico, os principais ativos com propriedades antioxidantes no extrato. Estes resultados confirmam a necessidade de se processar os extratos obtidos a partir de $R$. officinalis, visando a proteção e manutenção dos seus componentes ativos principais, que sofrem grande degradação ao entrar em contato com oxigênio devido a sua potente atividade antioxidante, mesmo quando armazenados a $20^{\circ} \mathrm{C}$. Essa queda indica que o processamento deve ser realizado no momento em que se realiza a extração dos compostos bioativos.

Com relação à comparação de ativos nas formulações líquidas e secas, observou-se que as formulações secas apresentaram maior concentração de ativos. Quando as formulações são comparadas quanto à proporção de adjuvantes, pode-se observar que, em geral, as maiores proporções de adjuvantes fornecem maiores quantidades de ativos. 
Tabela 4 - Quantificação por HPLC dos ativos presentes nas formulações.

\begin{tabular}{ccccc}
\hline Amostra & Ácido Cafeico (\%) & Ácido Rosmarínico (\%) & Carnosol (\%) & Ácido Carnósico (\%) \\
\hline EL-1 & $0,04 \pm 0,00$ & $4,11 \pm 0,03$ & $3,05 \pm 0,05$ & $2,96 \pm 0,15$ \\
EL-2 & $0,02 \pm 0,00$ & $4,35 \pm 0,07$ & $0,37 \pm 0,02$ & $0,37 \pm 0,00$ \\
F1L3 & $0,02 \pm 0,00$ & $4,12 \pm 0,03$ & $0,41 \pm 0,01$ & $0,30 \pm 0,00$ \\
F1L6 & $0,05 \pm 0,00$ & $4,48 \pm 0,02$ & $0,43 \pm 0,00$ & $0,33 \pm 0,00$ \\
F1P3 & $0,05 \pm 0,00$ & $4,28 \pm 0,02$ & $0,40 \pm 0,00$ & $0,22 \pm 0,00$ \\
F1P6 & $0,05 \pm 0,00$ & $4,50 \pm 0,03$ & $0,43 \pm 0,00$ & $0,23 \pm 0,00$ \\
F1LP3 & $0,04 \pm 0,00$ & $4,06 \pm 0,00$ & $0,43 \pm 0,00$ & $0,26 \pm 0,00$ \\
F1LP6 & $0,05 \pm 0,00$ & $4,19 \pm 0,01$ & $0,44 \pm 0,01$ & $0,25 \pm 0,00$ \\
F1L3-L & $0,04 \pm 0,00$ & $3,97 \pm 0,01$ & $0,46 \pm 0,00$ & $0,21 \pm 0,00$ \\
F1L6-L & $0,03 \pm 0,00$ & $3,83 \pm 0,02$ & $0,43 \pm 0,00$ & $0,22 \pm 0,00$ \\
F1P3-L & $0,03 \pm 0,00$ & $3,61 \pm 0,00$ & $0,46 \pm 0,00$ & $0,14 \pm 0,00$ \\
F1P6-L & $0,03 \pm 0,00$ & $3,69 \pm 0,05$ & $0,47 \pm 0,01$ & $0,14 \pm 0,00$ \\
F1LP3-L & $0,04 \pm 0,00$ & $3,88 \pm 0,02$ & $0,51 \pm 0,00$ & $0,17 \pm 0,00$ \\
F1LP6-L & $0,03 \pm 0,00$ & $3,47 \pm 0,01$ & $0,45 \pm 0,00$ & $0,14 \pm 0,00$ \\
\hline
\end{tabular}

-L: formulação líquida; EL-1: extrato liofilizado novo; EL-2: extrato liofilizado após 3 meses armazenado.

\section{CONCLUSÕES}

A presença de adjuvantes na secagem de formulações lipídicas influi consideravelmente no rendimento do processo de secagem, levando a uma maior recuperação de produto. $\mathrm{O}$ aumento da concentração de adjuvantes e a associação dos adjuvantes (lactose e proteína) na formulação também se mostrou eficiente, capaz de reduzir as perdas de produto durante a secagem.

Dispersões secas obtidas com lactose apresentaram maiores valores de atividade de água e umidade quando comparada à proteína do soro do leite, provavelmente devido a maior higroscopicidade. Porém, a associação desses dois adjuvantes também forneceu bons resultados de solubilidade, com menor atividade de água e umidade, o que é positivo para a estabilidade ao produto.

Quanto às características farmacotécnicas, as dispersões lipídicas obtidas neste trabalho, foram classificadas como de fluxo fraco a muito fraco, resultado comumente observado em produtos secos em spray dryer. Esta desvantagem pode ser superada pela adição de adjuvantes de fluidez como, por exemplo, dióxido de silício coloidal, talco, estearato de magnésio entre outros.

\section{REFERÊNCIAS}

ALMELA, L.; SÁNCHES-MUÑOZ, B.; FERNÁNDEZ-LÓPEZ, J. A.; ROCA, M. J.; RABE, V. Liquid chromatograpic-mass spectrometric analysis of phenolics and free radical scavenging activity of rosemary extract from different raw material. J. Chromatography A 1120, p. 221-229, 2006.

AULTON, M. E. Delineamento de formas farmacêuticas. 2 ed. Artmed, 2005.

BRASIL. ANVISA. Agência Nacional de Vigilância Sanitária. Resolução RE no 899, de 29 de maio de 2003. "Guia para validação de métodos analíticos e bioanalíticos". Diário Oficial da União, 2 de junho de 2003.

CALVO, P.; CASTAÑO, A. L.; LOZANO, M.; GONZÁLEZ-GÓMEZ D. Influence of the 
microencapsulation on the quality parameters and shelf-life of extra-virgin olive oil encapsulated in the presence of BHT and different capsule wall components. Food Research Int., v. 45, p. 256-261, 2012.

COUTO, R.O.; CONCEIÇÃO, E.C.; CHAUL, L.T.; OLIVEIRA, E.M.S.; MARTINS, F.S.; BARA, M.T.F.; REZENDE, K.R.; ALVES, S.F.; PAULA, J.R. Spray-dried rosemary extracts: Physicochemical and antioxidant properties. Food Chemistry, v. 131, p. 99-105, 2012.

CORTES-ROJAS, D.F.; OLIVEIRA， W.P. Physicochemical properties of phytopharmaceutical preparations as affected by drying methods and carriers. Drying Technology, v. 30, n. 9, p. 921-934, 2012.

CORTES-ROJAS, D.F.; SOUZA, C.R.F.; OLIVEIRA, W.P. Encapsulation of eugenol rich clove extract in solid lipid carriers. J. Food Engineering, v. 127, p.34-42, 2014.

COURRIER, H.M.; BUTZ, N.; ANDAMME, F. Pulmonary drug delivery systems: developments and prospects. Therap. Drug Carrier Systems, v. 19, p. 425-498, 2002.

EASTMAN, J.E.; MOORE, C.O. Cold water soluble granular starch for gelled food composition. US Patent, 4, 465-702, 1984.

FERNANDES, M.R.V. Obtenção, padronização e avaliação biológica de extratos secos a partir da espécie Psidium guajava L. pelo processo spray drying. $237 \mathrm{f}$. Tese. FCFRP/USP, 2013.

GENENA, A. K. Extração e caracterização do extrato de alecrim (Rosmarinus officinalis L.): Estudo de sua ação antioxidante. PPG em Engenharia de Alimentos/UFSC (Dissertação), 2005, 159p.

GORTZI, O.; LALAS, S.; CHINOU, I. TSAKNIS, J. Reevaluation of bioactivity and antioxidant activity of Myrtus communis extract before and after encapsulation in liposomes.
European Food Research and Technology, v. 226, p. 583-590, 2008.

LO, Y.; TSAI, J.; KUO, J. Liposomes and Disaccharides as Carriers in Spray-Dried Powder Formulations of Superoxide Dismutase. Journal of Controlled Release, v. 94, p. 259$272,2004$.

SECOLIN, V.A.; OLIVEIRA W.P.; SOUZA, C.R.F. Microencapsulação de catequinas de chá verde em carreadores lipossomais. In: Anais do XXXVI Congresso Brasileiro de Sistemas Particulados, 10 páginas, 2014.

SOUZA, C.R.F.; OLIVEIRA, W.P. Microencapsulation of Ketoprofen in Blends of Acrylic Resins by Spray Drying. Drying Technology, v. 30, n. 3, p. 263-275, 2012.

SOUZA, C.R.F.; SCHIAVETTO, I.A.; THOMAZINI, F.C.F.; OLIVEIRA, W.P. Processing of Rosmarinus officinalis Linné extract on spray and spouted bed dryers. Braz. J. Chem. Eng., v. 25, p. 59-69, 2008.

WELLWOOD, C.R.L.; COLE, R.A. Relevance of Carnosic Acid Concentrations to the Selection of Rosemary, Rosmarinus officinalis (L.), Accessions for Optimization of Antioxidant Yield. Journal of Agricultural and Food Chemistry, vol. 52, p. 6101-6107, 2004.

ZHANG, Y.; SMUTS, J.P.; DODBIBA, E.; RANGARAJAN, R.; LANG, J.C.; ARMSTRONG, D.W. Degradation study of carnosic acid, carnosol, rosmarinic acid, and rosemary extract (Rosmarinus officinalis L.) assessed using HPLC. J. Agricultural Food Chemistry, v. 60, p. 9305-9314, 2012.

\section{AGRADECIMENTOS}

Os autores agradecem a FAPESP pela concessão de bolsa de estudo ao primeiro (Proc.\# 2014/14692-4) e último autores (Proc. \# 12/03427-2) e auxílio financeiro a pesquisa Projeto JP (Proc. \# 11/10333-1). 